\title{
Dimensionamento de um landfarming para tratamento de borras oleosas utilizando critérios de um reator batelada
}

\author{
Scaling a landfarming treatment of oily sludge using criteria of a batch reactor. \\ C. E. M. Jerônimo' e W. G. C. M. Kitzinger² \\ 'Doutorado em Engenharia Quimica. Engenheiro de Processamento de Petroleo da Petrobras. Universidade Potiguar, Natal-RN, Brasil \\ ${ }^{2}$ Graduando em Engenharia de Petróleo e Gás, Universidade Potiguar, Natal-RN, Brasil
}

\section{Resumo}

Este trabalho apresenta um critério de dimensionamento de sistemas de landfarmings, que leva em consideração o modelo cinético da degradação dos poluentes presentes na borra oleosa, os níveis de velocidades envolvidos nas reações químicas e a adaptação da teoria do reator em batelada para o dimensionamento do terreno a ser instalado a infra-estrutura de tratamento, visto que esse assunto é considerado inédito na literatura e normas técnicas que direcionam os modelos de dimensionamento desses sistemas. A metodologia empregada no desenvolvimento desse trabalho implicou na análise interpretativa de textos e imagens presentes em artigos científicos, livros, e trabalhos de conclusão de curso. Assim como análise da Norma ABNT NBR 13894 de 1997 para tratamento no solo (landfarming). Foram obtidos os dados cinéticos e considerados ajustes matemáticos para cinéticas de primeira e segunda ordem para as reações analisadas. O critério proposto, com a utilização dos princípios de funcionamento de um reator batelada aplicado a um sistema landfarming apresentou-se como um método aceitável e consiste num avanço ao estado da arte das metodologias para um dimensionamento seguro e eficiente para esse tipo de sistema.

Palavras-chave: Landfarming, Reator batelada, Borras oleosas

\begin{abstract}
This paper presents a criterion for sizing systems landfarmings, which takes into account the kinetic model of the degradation of pollutants in oily sludge, levels of speeds involved in chemical reactions and adaptation of the theory of batch reactor for the sizing of the ground be installed infrastructure for treatment, since this subject is considered unprecedented in the literature and technical standards that guide the design models of these systems. The methodology used in the development of this work involved the interpretative analysis of texts and pictures present in scientific articles, books, papers and course completion. As analysis of ABNT NBR 138941997 for soil treatment (landfarming) . Kinetic data were obtained and considered for kinetic mathematical adjustments first and second order reactions analyzed. The proposed criterion, using the principles of operation of a batch reactor applied to a system Landfarming presented as an acceptable method and consists in advancing the state of the art design methodologies for safe and efficient for this type of system..
\end{abstract}

Keywords: Landfarming, Batch reactor, Oily waste 


\section{INTRODUÇÃO}

Diferentes etapas da exploração e refino do petróleo resultam na geração de borras oleosas que possuem em sua composição substâncias que imprimem periculosidade aos mesmos, e que, caso sejam dispostas de forma inadequada podem vir a contaminar o solo, e consequentemente podem impactar a biota da área, atingir os lençóis freáticos, rios e lagos da região, agredindo diretamente ou indiretamente a saúde humana e gerando custos ambientais, sociais e monetários muito altos ${ }^{1}$.

O solo poluído torna-se uma ameaça ao meio ambiente por apresentar substâncias tóxicas, entre estas substâncias podemos citar os hidrocarbonetos aromáticos e os metais pesados ${ }^{2}$. Logo, é de fundamental importância a adoção de técnicas de tratamento seguras e eficientes para o tratamento dos resíduos da indústria de Petróleo.

Os resíduos como as borras de petróleo eram, até um passado bastante recente, acondicionados em tanques ou dispostos em aterros, de forma praticamente aleatória, mesmo em países desenvolvidos, em função da inexistência, até então, de uma legislação ambiental mais específica ${ }^{3}$. Entretanto buscam-se soluções para o problema, realizando a remoção das substâncias tóxicas das borras oleosas com a execução de tratamento para estas borras. Dentre estes tratamentos existe a técnica denominada de landfarming, que apresenta um baixo custo e uso mais amplo no Brasil.

A técnica do landfarming consiste no espalhamento do material oleoso sobre o solo preparado e a incorporação do óleo na camada arável até que a ação microbiana degrade os componentes presentes na borra (com auxílio do controle da umidade, $\mathrm{pH}$ e nutrientes no solo). Esse processo sofre influência direta da taxa de atividade dos microrganismos responsáveis pela biodegradação dos resíduos ${ }^{1}$. Quando se consideram resíduos industriais passíveis de biodegradação, a disposição final destes pode ser realizada, de maneira segura e econômica, através do uso de landfarming ${ }^{4}$. O solo possui, naturalmente, diversos microrganismos com atividades metabólicas bastante variadas. Sendo assim, uma das maneiras mais simples de se proceder no tratamento de um resíduo consiste em misturá-lo ao solo e deixar que a flora microbiana nativa atue ${ }^{5}$.

Em períodos de chuvas intensas não se aplica resíduos nos landfarmings abertos, uma vez que o processo de tratamento é aeróbio e sendo assim o excesso de umidade faz com que aconteça a anaerobiose $^{6}$. Logo, uma recomendação que a maioria dos órgãos ambientais tem feito é para aprovação apenas de sistemas cobertos.

A biodegradação de resíduos de hidrocarbonetos depende da estrutura química do composto, da quantidade e frequência da disposição do resíduo no solo, e das características físicas, químicas e biológicas do solo e requer ampla capacidade metabólica, dependendo, portanto, da composição da comunidade microbiana e de sua adaptação aos hidrocarbonetos presentes no resíduo ${ }^{7}$. Logo, a cinética de biodegradação deve ser conhecida para o correto dimensionamento e definição dos períodos a serem utilizados no tratamento adequado das borras oleosas.

Esse tratamento, por ser um processo de baixo custo para tratamento de grandes volumes de resíduos, tem sido utilizado com sucesso por décadas, no biotratamento de substâncias derivadas do petróleo e que muitas são liberadas pelas indústrias de refino de óleo ${ }^{8}$. Entretanto, nem sempre o dimensionamento é feito de uma forma correta, ou adota mecanismos técnicos para a escolha dos dimensionais e planos de manejo.

As questões no dimensionamento, por exemplo, não consideram pontos como: se são usados somente resíduos pesados ou óleo cru no sistema de landfarming, se a porção residual resistente ao metabolismo microbiano é bem mais elevada que quando são adicionados resíduos de médio peso molecular, dentre outros efeitos cinéticos das reações de degradação9.

Logo, para a utilização do sistema landfarming, quase sempre se tem o emprego de grandes áreas, para compensar a ausência dos critérios de dimensionamento e compensar com elevados fatores de segurança. E situada bem longe do abastecimento de água potável e o solo não pode ser permeável. Depois que o óleo for degradado, o solo pode ser utilizado para o cultivo de uma grande variedade de árvores e plantas herbáceas, incluindo gramíneas ${ }^{10}$.

Dessa forma, este trabalho apresenta um critério de dimensionamento de sistemas de landfarmings, que levam em consideração o modelo cinético da degradação dos poluentes presentes na borra oleosa, os níveis de velocidades envolvidos nas reações químicas e a adaptação da teoria do reator em batelada para o dimensionamento do terreno a ser instalado a infra-estrutura de tratamento, visto 
que esse assunto é considerado inédito na literatura e normas técnicas que direcionam os modelos de dimensionamento desses sistemas.

\section{MATERIAIS E MÉTODOS}

Considerando o objetivo deste artigo, realizou-se uma pesquisa qualitativa de caráter descritiva. Nesse tipo de pesquisa qualitativa os resultados dependem também do pesquisador, de sua compreensão e interpretação dos dados. Uma pesquisa qualitativa propõe que os dados devem ser analisados mediante um contexto, observando a lógica, a coerência e a ligação entre as informações ${ }^{11-12}$.

A pesquisa de campo usada como base para elaboração deste artigo é composta por um Estudo de Caso que permite um levantamento qualitativo aprofundado sobre a unidade que se investiga. Para isso o pesquisador pode se valer de diversas técnicas de coleta de dados, reunindo um maior número de informações, de forma a compreender todas as variáveis da unidade analisada ${ }^{12}$.

O objeto de estudo deste artigo foi a criação de um critério de dimensionamento de landfarmings através da teoria de dimensionamento de reatores bateladas ${ }^{13}$, que, além de ser o núcleo dos modelos permitirá o desenvolvimento de uma abordagem segura aos dados secundários utilizados.

Ainda como fontes de pesquisas, a coleta de dados valeu-se de análise documental, importante principalmente pelo acesso a alguns trabalhos acadêmicos, sobretudo, aos dados secundários da cinética de decomposição de agentes químicos apresentada por Silva ${ }^{14}$.

A análise documental como uma consulta a documentos internos da instituição ou setor pesquisado, a fim de coletar dados que facilitem o entendimento do que está sendo pesquisado ${ }^{12}$.

A metodologia empregada no desenvolvimento desse trabalho implicou na análise interpretativa de textos e imagens presentes em artigos científicos, livros, e trabalhos de conclusão de curso. Assim como análise da Norma ABNT NBR 13894 de 1997 para tratamento no solo (landfarming) ${ }^{11}$. Foram obtidos os dados cinéticos e considerados ajustes matemáticos para cinéticas de primeira e segunda ordem para as reações analisadas.

\section{RESULTADOS E DISCUSSÃO}

\section{I. Descrição da téCnICa Landfarming}

O landfarming é um sistema de tratamento frequentemente escolhido para tratamento das borra oleosas de petróleo porque é de custo baixo e alto potencial de sucesso ${ }^{2}$.

Este processo utiliza microorganismos do solo para tratamento dos resíduos industriais orgânicos biodegradáveis. Para isto, é mantida uma população microbiana aderida nas partículas do solo, que é colocada em contato com o resíduo a ser degradado².

Ou seja, a biodegradação microbiana, que é o mecanismo primário de eliminação dos poluentes orgânicos do ambiente, compõe a base deste tratamento ${ }^{14}$.

A utilização do solo para o tratamento de resíduos teve início na Europa no final do século XIX com a técnica de irrigação de áreas cultivadas com águas residuárias sanitárias. No início dos anos 50, o processo de tratamento no solo despertou interesse nas empresas de refino de petróleo dos Estados Unidos, sendo as primeiras a desenvolver e praticar o tratamento no solo para seus resíduos e, para este processo de tratamento específico, elas deram o nome de landfarming ${ }^{17}$.

No inicio da década de 1950 não havia disposições tecnológicas para o tratamento e /ou reaproveitamento de borras oleosas geradas pela indústria do petróleo. Sendo assim o destino destas eram lagoas ou diques. Em alguns caos, a borra era simplesmente descartada em trincheiras, valas ou colocadas em tambores e depois enterrados, sem nenhum preparo da área, e com isso aumentando os riscos ambientais que este material traria ao meio ambiente, ou seja, sem uma gestão ambiental adequada. Com o passar dos anos, estes locais tornaram-se o principal foco de contaminação do solo e, posteriormente, devido à liquefação da borra e a percolação e infiltração da águas pluviais, acarretou sua migração e de seus contaminantes para o lençol freático. As principais contaminações originadas são de hidrocarbonetos alifáticos (TPH), hidrocarbonetos policíclicos aromáticos (PAH) e BTEX (benzeno, tolueno, etilbenzeno e xilenos) ${ }^{17}$. 
Outro país que desenvolveu o tratamento no solo foi a Alemanha, sendo que no ano de 1960 já estavam em operação 60 áreas para tratamento de resíduo no solo, e até o ano de 1987 esse processo ocupava uma área de 20.000 hectares $^{14}$.

No Brasil, o landfarming foi introduzido pela Petrobras quando, no ano de 1982, colocou em funcionamento uma unidade na Refinaria Henrique Lage, na cidade de São José dos Campos, Estado de São Paulo. Desde então, a Petrobras iniciou a implantação de landfarming em outras refinarias ${ }^{15}$.

Esta tecnologia está basicamente direcionada ao tratamento de resíduos industriais classificados segundo a NBR 10.004 - Classificação de Resíduos - como Classe I (perigoso) e Classe II (não inerte) ${ }^{11}$. Na Figura 1 podemos observar a disposição básica do terreno de landfarming.

\section{Typical Landfarming Operation}

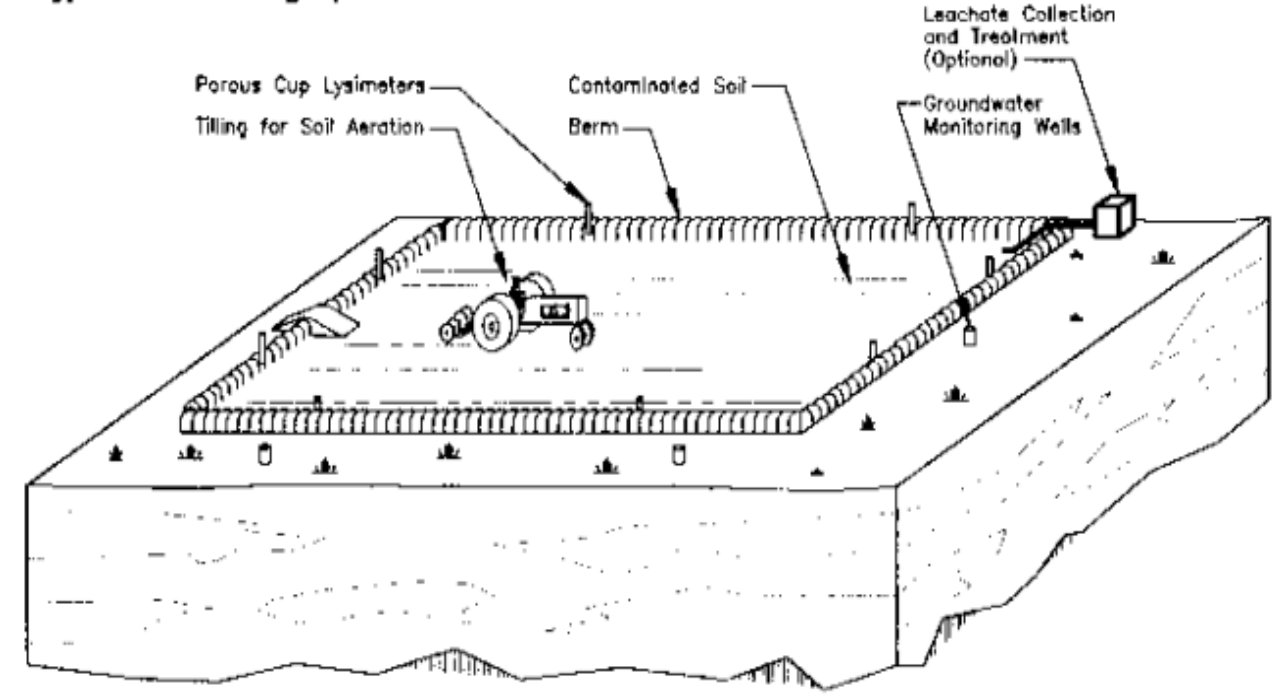

Figura 1: Esquema de um terreno landfarming.

Fonte: http://www.nmenv.state.nm.us/ust/images/remed-4.gif

A metodologia do landfarming objetiva, através do uso de técnicas agrícolas, tais como a aeração mecânica e a adubação química, aumentar a ação decompositora de microrganismos presentes no solo, para então tratar resíduos que contenham frações sólidas e aquosas in situ. O espalhamento da borra oleosa, sobre o solo e a incorporação dos mesmos na camada arável, influencia diretamente a taxa de atividade dos microrganismos responsáveis pela biodegradação dos resíduos. ${ }^{1}$ Os landfarmings, geralmente, são formados por áreas com cerca de 0,5 - 1,0 ha, denominadas células de biodegradação ${ }^{18}$.

Quando os resíduos são adicionados ao solo, acontecem os seguintes processos: degradação biológica, incorporação na matriz do solo, volatilização, percolação e lixiviação superficial. Além disso, muitos processos bioquímicos acontecem no "landfarming", resultando na conversão de carbono a dióxido de carbono. O carbono remanescente é incorporado à massa celular e fixado ao solo como matéria orgânica natural melhorando assim a sua estrutura e fertilização ${ }^{1}$.

Muitos destes resíduos possuem características potencialmente perigosas à saúde humana e ao meio ambiente, além de existir uma tendência mundial para o aumento da demanda e do consumo de derivados de petróleo nos próximos anos ${ }^{1}$.

As taxas de aplicação dos resíduos não devem ser tão baixas que exijam grandes áreas e nem tão altas que sobrecarregue os microorganismos do solo fazendo decair a taxa de degradação ${ }^{5}$.

Os resíduos oleosos e graxas apresentam uma alta tratabilidade no solo. Ressalta-se que a elevada capacidade de tratamento no solo não significa ausência de risco de poluição ambiental, entretanto, como parte da gordura presente nos resíduos mencionados são de origem animal, acredita-se que os mecanismos de biodegradação no solo sejam apropriados ${ }^{19}$.

O landfarming oferece riscos de poluição se a estabilização da borra oleosa não for completa podendo contaminar os lençóis freáticos e causar problemas de saúde, visto que os compostos orgâni- 
cos na borra de petróleo incluem alguns aromáticos e poliaromáticos, além de compostos inorgânicos que podem conter metais pesados ${ }^{16}$.

A norma ABNT NBR ISO 13.894 (Tratamento no Solo - Landfarming) estabelece que o projeto, construção, operação e manutenção da unidade devem ser realizados de forma a elevar ao máximo a degradação, a transformação e/ou imobilização de contaminantes da camada reativa do solo.Esta norma preconiza, ainda, alguns requisitos relativos à topografia, solos e geologia, recursos hídricos, vegetação, vias de acesso, distância a núcleos populacionais, entre outros aspectos.Portanto, todo um preparo da área é exigido, a fim de que se reduzam, ao máximo, os riscos de contaminação de lençóis freáticos por lixiviação de poluentes ${ }^{15}$.

Para obter melhor desempenho no landfarming a toxicidade deve ser avaliada, além do nível de metais pesados presentes nos resíduos oleosos, considerando que os metais pesados residuais do processamento do petróleo permanecerão nas lamas oleosas, pois não são decompostos pelos microorganismos no landfarming, sendo assim permanecem no resíduo como um passivo ${ }^{2}$. Por isso as células de biodegradação recebem uma camada compactada de argila para evitar a lixiviação de metais e compostos orgânicos poluentes das águas subterrâneas, um sistema de drenagem e uma camada reativa de solo, preparada para receber o resíduo ${ }^{18}$. Na Figura 2 pode-se observar as camadas do terreno.

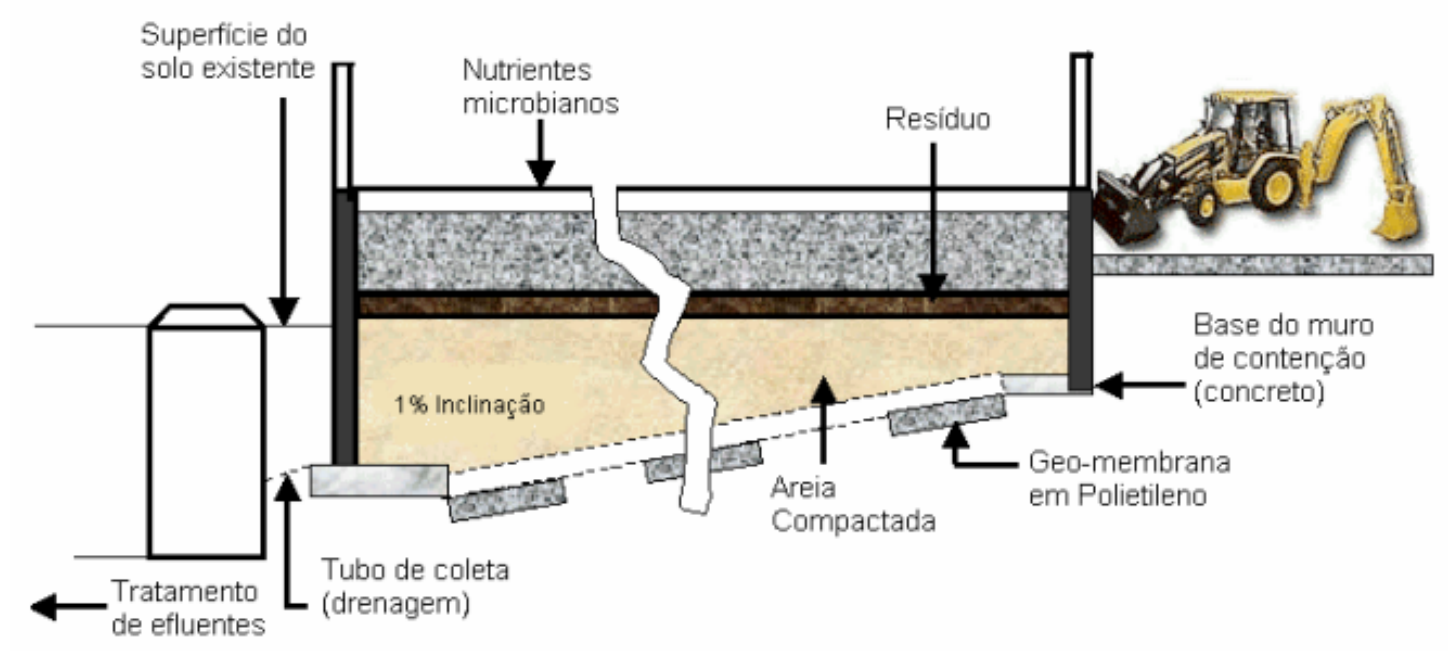

Figura 2: Demonstração da camada de nutrientes microbianos, resíduo e areia compactada. Fonte: http://www.responsiblebusiness. eu/download/attachments $/ 2916488 /$ Picture7.png?version=1\&modificationDate $=1277880153000$

As substâncias não biodegradáveis tais como metais pesados, sais e areia, o "landfarming" age apenas como acumulador. Nesse caso, se a célula vier a ficar saturada dessas substâncias, deve-se raspar a camada reativa $(15-20 \mathrm{~cm})$ e enviá-la para um aterro industrial ${ }^{17}$. Compostos clorados ou nitrogenados são, em geral, difíceis para degradar ${ }^{14}$.

As técnicas operacionais envolvem adição de nutrientes, umidificação, aeração e correção do $\mathrm{pH}$ do solo. Os macronutrientes adicionados são compostos a base de nitrogênio, fósforo e potássio sob a forma de fertilizantes comerciais e/ou uréia. A correção do $\mathrm{pH}$ do solo pode ser feita pela adição de óxidos de cálcio e magnésio. A aeração do solo é viabilizada através de arado acoplado a trator ${ }^{15}$.

Em landfarming com solo argiloso e compacto, a adição de materiais de baixo custo e fácil disponibilidade como serragens, casca de algodão e palhadas podem trazer efeitos benéficos no processo de biodegradação aeróbia, facilitando a mistura e revolvimento do solo/resíduo, auxiliando a drenagem de águas superficiais e retendo a umidade do solo em períodos de secas ${ }^{18}$.

$\mathrm{Na}$ camada reativa ou zona de tratamento, a água superficial é controlada e monitorada para evitar carência de oxigênio ${ }^{18}$. Na Figura 3 temos um terreno landfarming real, em modelo aberto e na Figura 4 no modelo coberto. 


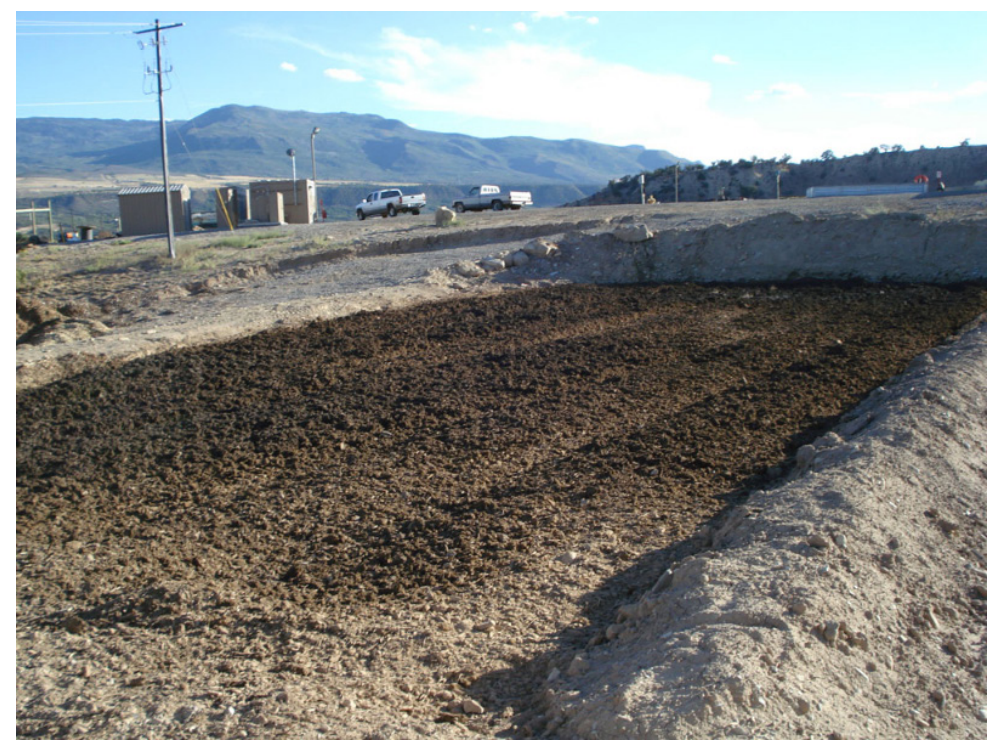

Figura 3: Terreno de landfarming real.

Fonte: http://www.rimlabenvironmental.com/wp-content/gallery/landfarming/land-farming-after.jpg

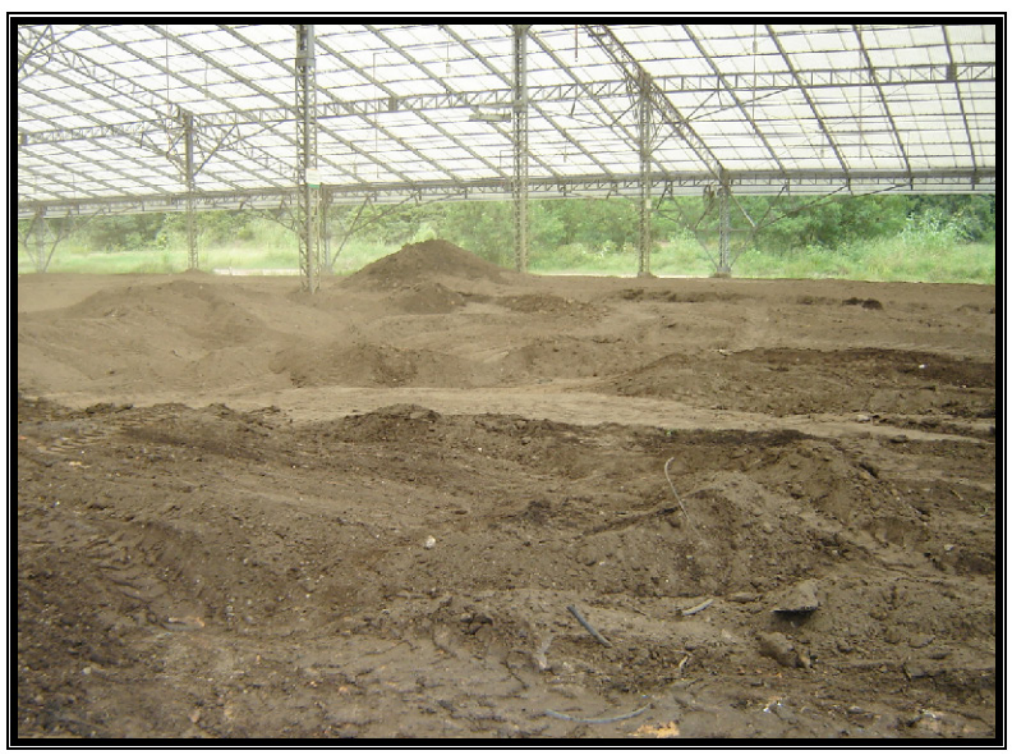

Figura 4: Landfarming coberto. Fonte: Silva( 2009)

A seleção do local, além de ser fundamental para garantir a eficiência do processo é importante para garantir a segurança e proteção ambiental. Locais com falhas geológicas, alta permeabilidade, aqüíferos utilizados para abastecimento de água, nível alto de águas subterrâneas, susceptibilidade a erosão, locais pantanosos, declividade superior a 5\%, são desaconselháveis para implantação deste tipo de tratamento ${ }^{14}$.

O conhecimento da volatilidade dos contaminantes propostos para o tratamento por landfarming é muito importante, devido ao fato dos constituintes voláteis tenderem a evaporar do local de tratamento mais rapidamente do que serem biodegradados pelos micro-organismos, particularmente durante as operações de revolvimento ou aragem. O controle das emissões de compostos orgânicos voláteis pode ser requerido e pode envolver a captura dos vapores emitidos para a atmosfera, que 
devem, então, ser encaminhados para processos de tratamento apropriados. Uma simples cobertura da área com um plástico pode funcionar como um controle para o escape dos gases, bem como uma estufa para cobrir a área ${ }^{16}$.

Os landfarmings são geralmente descobertos e,por isto, são suscetíveis a fatores climáticos, incluindo chuva, neve, vento e temperatura ambiente. Quando ocorre precipitação diretamente sobre a área, o teor de umidade do solo aumenta e, ao secar, pode ocorrer erosão. Por outro lado, em períodos de seca, o teor de umidade pode ficar abaixo daquele requerido pelos microorganismos ${ }^{20}$.

Dentre as vantagens do tratamento temos: Ser relativamente simples de projetar e executar; ter pequeno tempo de tratamento (normalmente 6 meses a 2 anos sob condições ótimas); ser efetivo em componentes orgânicos com taxas de biodegradação lentas; ter baixo custo, da ordem de R $\$ 140,00$ o metro cúbico ${ }^{19}$. E como desvantagens, tem-se: redução de concentração maior que $95 \%$ e concentrações constituintes menos que 0,1 ppm são muito difíceis de alcançar; pode não ter efetivo para concentrações constituintes altas (maior que 50.000 ppm de hidrocarboneto de petróleo totais); a presença de concentrações de metais pesados significantes (maior que 2,500 ppm) pode inibir crescimento microbiano; os constituintes voláteis tendem a evaporar em lugar de biodegradar durante o tratamento ${ }^{19}$.

\subsection{Dados Secundários da Biodegradação}

Para o desenvolvimento do modelo matemático a ser utilizado no dimensionamento de um sistema landfarming, tomou-se como base os resultados obtidos por Silva ${ }^{14}$, cujo monitoramento dos parâmetros cinéticos ocorreu por metodologia padronizada considerando elementos como:

Amostragem do solo: Para verificar o desempenho do bioprocesso foi estabelecida uma freqüência mensal de amostragem da camada reativa do solo do landfarming seguindo a norma ABNT NBR ISO 10.007 (Amostragem de Resíduos, 2004) ${ }^{14}$.

Analise física: As determinações físicas do solo, em termos de umidade e $\mathrm{pH}$ foram determinadas no Laboratório de Microbiologia Aplicada à Indústria do Petróleo da Universidade Federal do Rio de Janeiro ${ }^{14}$.

Umidade: As amostras de solo foram transferidas para placas de Petri previamente taradas; após a pesagem, o conjunto foi levado para estufa (Biomatic, modelo 1451 ) a $80 \pm 1^{\circ} \mathrm{C}$, por quarenta e oito horas ${ }^{14}$.

pH: Em um becker de capacidade de $50 \mathrm{~mL}$ foram transferidos $10 \mathrm{~g}$ de solo medido em balança analítica (ADA, 210/L). Determinou-se o $\mathrm{pH}$ em potenciômetro (DIGIMED, DM-20) previamente calibrado com soluções tampão de $\mathrm{pH}=4,00$ e 7,00 ${ }^{14}$.

Análise química: As seguintes determinações analíticas foram realizadas: teor de Carbono Orgânico Total (COT), Nitrogênio Total e Fósforo; Hidrocarbonetos Totais do Petróleo (HTP); Hidrocarbonetos Policíclicos Aromáticos (HPA); e Elementos Químicos (Antimônio, Arsênio, Bário, Berílio, Cádmio, Chumbo, Cobre, Cromo Total, Ferro Total, Manganês, Mercúrio, Níquel, Prata, Selênio, Vanádio, e Zinco).

Hidrocarbonetos Totais do Petróleo (HTP): A quantificação de Hidrocarbonetos Totais do Petróleo (HTP) foi realizada pelo método USEPA 8015B (Nonhalogenated organics using GC/FID) de cromatografia gasosa acoplada ao detector por ionização de chamas.

Hidrocarbonetos Policíclicos Aromáticos (HPA): Para determinação de Hidrocarbonetos Policíclicos Aromáticos (HPA) foram utilizadas as metodologias USEPA 3540C (Soxhlet Extraction) e USEPA 3630C (Silica Gel Column Cleanup) e USEPA 8270C (Semivolatile Organic Compounds by Gas Chromatography/ Mass Spectrometry - GC/MS). 


\subsection{Cinética Química da Biodegradação}

O mecanismos de biodegradação pode ser expresso por diferentes modelos matemáticos, entretanto, a degradação de componentes paralelo convergindo para a produção final de gás carbônico, como um dos mais aceitos e propagados para representar as reações intermediárias da biodegradação referente as diferentes fases do metabolismo microbiano. No esquemático abaixo é mostrado a forma do modelo utilizado no presente trabalho:

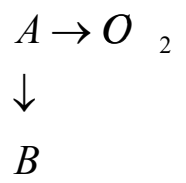

Onde as reações $\left(A \rightarrow C O_{2}\right.$ são representadas pela constante $k_{1} ; A \rightarrow B$, constante $k_{2} e$ $\mathrm{B} \rightarrow \mathrm{CO}_{2}$ pela constante $\mathrm{k}_{3}$ ).

Considerando a modelagem matemática utilizada por Napoleão e colaboradores ${ }^{20}$, pode-se ajustar esse modelo pela equação:

$$
y=y_{0}+A_{1} e^{-\left(x / t_{1}\right)}+A_{2} e^{-\left(x / t_{2}\right)}
$$

Onde:

$\mathrm{y}=$ relação da concentração pontual e a final para o parâmetro monitorado.

$$
A_{1}=\frac{k_{1}-k_{3}}{k_{1}+k_{2}-k_{3}}, A_{2}=\frac{k_{2}}{k_{1}+k_{2}-k_{3}}, t_{1}=\frac{1}{k_{1}+k_{2}} e t_{2}=\frac{1}{k_{3}}
$$

O ajuste desses parâmetros pode ser obtido por meio da aplicação nas ferramentas do Statistica 7.0, para uma função exponencial dec2.

Considerando os dados obtidos por Silva ${ }^{14}$ foi possível o ajuste dos parâmetros $\mathrm{k}_{1}, \mathrm{k}_{2} \mathrm{e}_{3}$. Obtendo-se um coeficiente de correlação de $22 \%$ para os dados de HTP e $16 \%$ para os dados de HPA, o que demonstra que tal modelo não teve um ajuste satisfatório. Diante desse quadro ajustou-se ao padrão de uma cinética simples considerada pseudo-primeira ordem, inclusive simplificando todo o processo de dimensionamento futuro.

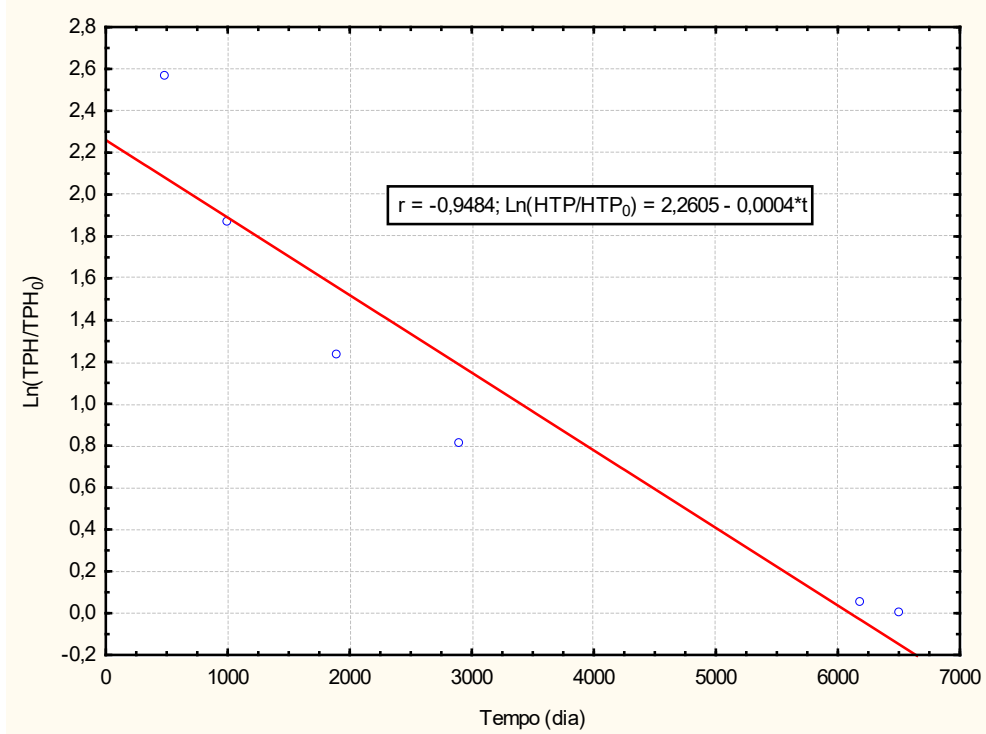


Figura 5: Ajuste Cinético dos Dados de Silva (2009) para decomposição de HTP

Na Figura 5 são apresentados os resultados obtidos para o ajuste de tais dados, e determinação da constante de velocidade especifica da reação $\left(K_{1}\right)$. Nesse caso optou-se pelo uso apenas da variável HTP, pois o propósito do dimensionamento e funcionalidade do equipamento leva a biodegradação de todos os componentes presentes no sistema. $\mathrm{O}$ ajuste da cinética de pseudo-primeira ordem teve um coefienciente de correlação superior a $94 \%$, considerado aceitável para a metodologia utilizada. O valor para a constante cinética $\mathrm{K}_{1}$ foi de $0,0004 \mathrm{dia}^{-1}$. E posteriormente terá sua aplicação descrita no modelo.

Em outros cenários, para aplicação da referida metodologia é recomendada a realização de ensaios de tratabilidade, com a obtenção de constantes específicas para os resíduos e solos a serem utilizados no sistema. Na ausência desses, a adoção dos valores aqui apresentados é uma boa referência na minimização dos erros de configuração dos sistemas.

\subsection{Dimensionamento do Landfarming}

Com base nos dados cinéticos de monitoramento da concentração de Hidrocarbonetos Totais do Petróleo (HTP) durante o período de tratamento será considerada a adaptação para a teoria de reator em batelada. Logo, a filosofia de funcionamento que é pretendida ao dimensionamento consiste em um o terreno da melhor cuja altura máxima do equipamento deve ser de $0,45 \mathrm{~m}$ (camada reativa) com erro de $\pm 0,05 \mathrm{~m}$. Dessa forma, o desafio é dimensionar o volume da referida área, como mostrado na Figura 6. Sendo assim temos a equação: $\mathrm{V}=\mathrm{x}$. y . 0,45 (onde $\mathrm{V}$ é o volume de área de tratamento do landfarming, e x/y os dimensionais de área do equipamento, em $\mathrm{m}$ ). Considerando o critério para o tratamento de 1 tonelada de solo contaminado por dia.

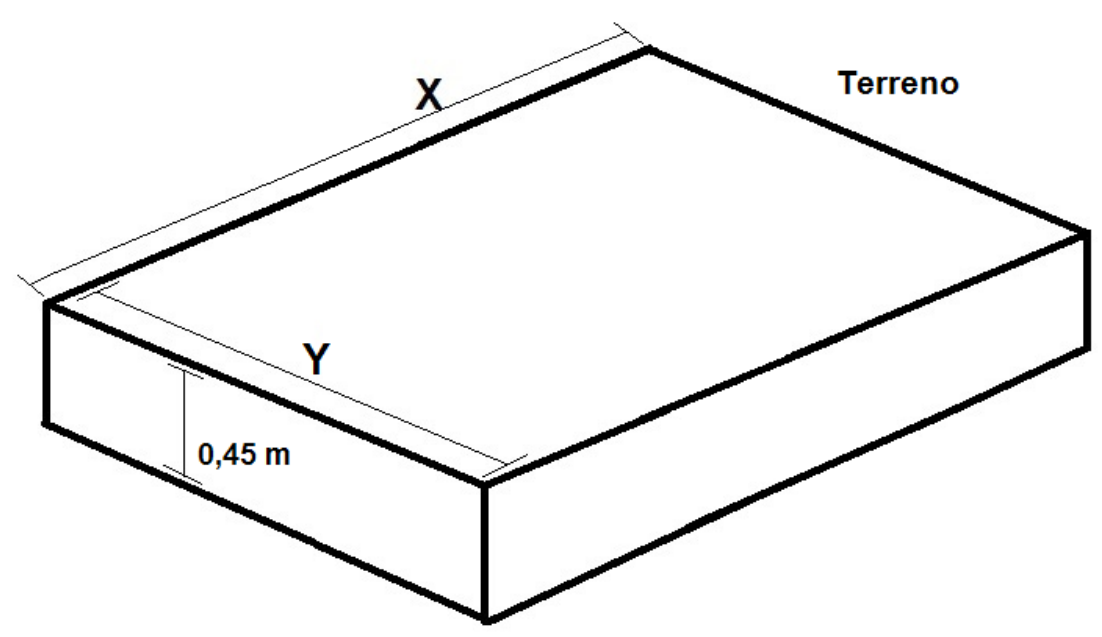

Figura 6: Medidas de terreno Landfarming. Fonte: Autor.

Com base na teoria do reator em batelada ${ }^{13}$, temos que para a decomposição do poluente (ora representado pela concentração $\left.\mathrm{Ca}\left[\mathrm{kg} \cdot \mathrm{m}^{-3}\right]\right)$, a conversão deste em gás carbônico pode ser mensurada pela seguinte expressão:

$$
X=\frac{C a_{0}-C a}{C a_{0}} \text { ou } C a=C a_{0}(1-X)
$$

Onde $\mathrm{Ca}_{0}$ é a concentração inicial e Ca é a concentração final [kg. $\left.\mathrm{m}^{-3}\right]$. A concentração se refere no caso, ao valor de TPH. 
Considerando que as reações processadas foram definidas como de pseudo-primeira ordem, o cálculo do volume de um reator batelada, ou no caso estudo do landfarming pode ser definida como sendo:

$$
V=\int_{0}^{X} C a_{0} \frac{d X}{d R a}=\int_{0}^{X} C a_{0} \frac{d X}{K_{1} C a(1-X)}=C a_{0} \int_{0}^{X} \frac{d X}{K_{1} C a(1-X)}
$$

Utilizando-se de métodos matemáticos de resolução para a equação (4), o volume pode ser estimado pela relação para a regra do trapézio, como sendo:

$$
V=\frac{\Delta X}{3}\left[\frac{C a_{0}}{-K_{1}}+\frac{4 C a_{0}}{-K_{1}\left(1-X_{1}\right)}+\frac{C a_{0}}{-K_{1}\left(1-X_{2}\right)}\right]
$$

Onde $\mathrm{X}$ e $\Delta \mathrm{X}$ são configurados nos critérios adotados no ato do dimensionamento. Sendo: $\mathrm{X}$, $\mathrm{X}_{1}$ e $\mathrm{X}_{2}$ significam respectivamente o ponto inicial, o ponto médio e o ponto final da faixa de eficiência requerida para o projeto, considerada por um terço da regra de Simpson. O dimensionamento de X deverá ser realizado com base nos padrões de descarte legal (legislação especifica) para o nível de tratamento requerido. Por exemplo, se o alvo de descarte é uma concentração de 500 ppm de HTP, e o solo contaminado apresenta um nível de 5.000 ppm de HTP, o objetivo do tratamento é $90 \%$ de remoção. Logo, deve-se estratificar o modelo para: $\mathrm{X}_{1}=0,45 ; \mathrm{X}_{2}=0,90$. Considerando intervalos regulares. Essa representação é demonstrada na Figura 7.

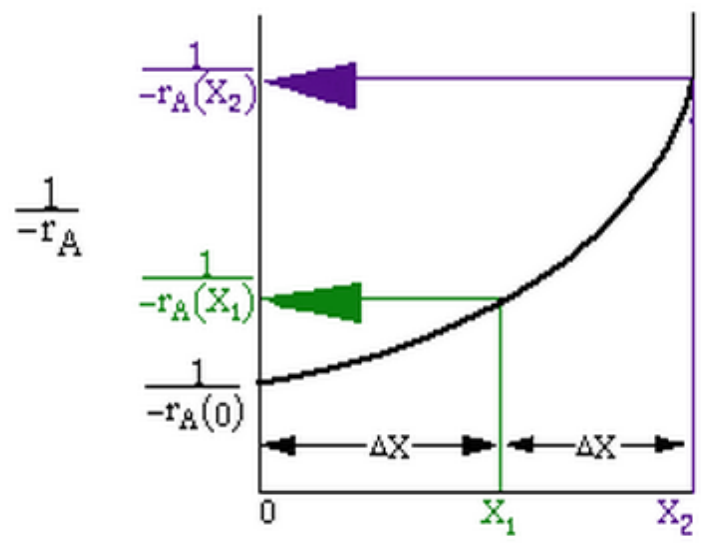

Figura 7: Um terço da regra de Simpson.

Fonte: http://www.umich.edu/ elements/course/lectures/two/index.htm

Fazendo a junção do critério dimensional da Figura 6, e estabelecendo uma relação que o comprimento é o dobro da largura do landfarming (critério sugerido pela facilidade de locomoção de tratores, seccionamento de células e facilidade de disponibilidade de áreas nesse formato), pode-se determinar a largura do equipamento (y) como sendo:

$$
y=\sqrt{0,3 \Delta X\left[\frac{C a_{0}}{-K_{1}}+\frac{4 C a_{0}}{-K_{1}\left(1-X_{1}\right)}+\frac{C a_{0}}{-K_{1}\left(1-X_{2}\right)}\right]}
$$


Esse modelo deve ser aferido para a vazão mássica de material a ser processado por dia, considerando as devidas correções de densidade requerida.

Diante desse equacionamento pode-se simplificar a metodologia de dimensionamento de um landfarming como sendo:
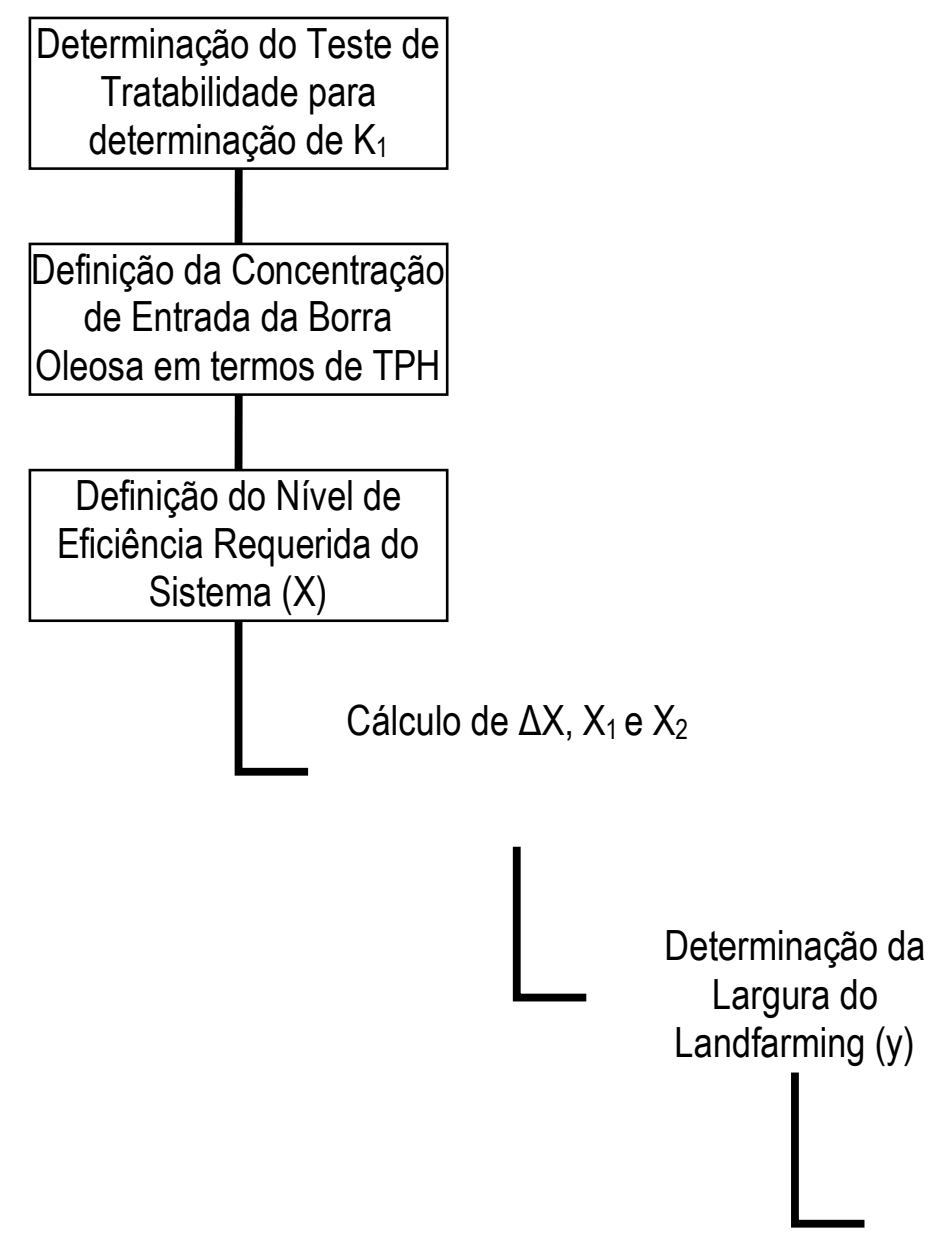

\section{Determinação do \\ Comprimento do} Landfarming (Dobro

Figura 8: Esquemático do Método de Cálculo Proposto

Fonte: Autor

Considerando os dados obtidos pelos dados de Silva ${ }^{14}$, um landfarming típico para o tratamento de 1 tonelada por dia (divididos em 15 células), considerando uma degradação de 0,0004 dia ${ }^{-1}$ e uma remoção de $90 \%$, teria a necessidade de uma área de $55 \mathrm{~m}$ de largura por $110 \mathrm{~m}$ de comprimento, adotando-se a metodologia acima proposta. Considerando o exemplo da área trabalhada por Silva ${ }^{14}$, em uma estrutura real, os dados demonstraram uma variação de apenas $5 \%$ do dimensional e níveis de eficiência obtida no sistema estudado, o que atesta como adequada a metodologia proposta nesse trabalho.

\section{CONCLUSÃO}

Concluí-se que a metodologia proposta, com a utilização dos princípios de funcionamento de um reator batelada aplicada a um sistema landfarming apresenta como um método aceitável e consiste 
num avanço ao estado da arte das metodologias para um dimensionamento seguro e eficiente para esse tipo de sistema.

Observou-se, ainda, que landfarming é uma boa prática de gestão, possuindo resultado satisfatório com custo pequeno e sendo uma prática ecologicamente correta para tratar resíduos os resíduos perigosos gerados na refinaria se tomados os devidos cuidados. Logo, havendo uma disposição dos resíduos gerenciada de forma cuidadosa e correta, o tratamento mostra-se como uma grande vantagem por ser simples e funcional, difundida pelo mundo e bem aplicada as condições do Brasil por 31 anos.

Entretanto, deve-se ter cautela na aplicação, pois existe o risco de se sobrecarregar os microorganismos do solo e consequentemente gerarem um passivo ambiental na área, ocasionando a poluição do solo. Caso, exista condições tecnológicas e econômicas, deve-se utilizar o landfarming como método alternativo ${ }^{21}$.

Dessa forma, esse trabalho se mostra de grande validade para o calculo do terreno landfarming se esse for o método escolhido pela empresa petrolífera que almeja um tratamento a baixo custo, tomando-se os devidos cuidados. Uma vez que o dimensionamento do landfarming nunca foi antes debatido, a prática errônea do super dimensionamento de terrenos vem acarretando custos desnecessários ou o uso de pequenos terrenos, sobrecarregando a ação dos microorganismos atuantes, proporcionando poluição ambiental e prejuízos para a companhia. O presente cálculo se faz necessário para uso adequado deste tratamento.

\section{REFERÊNCIAS}

1 - SOARES. C. E. Gestão de resíduos perigosos em refinarias de petróleo. Universidade do Estado do Rio de Janeiro, Rio de Janeiro, Março de 2009.

2 - GUARACHO, V. V. Remediação eletrocinética de chumbo e Niquel em solos de landfarming de refinaria. Universidade Federal do Paraná, Curitiba, 2005.

3 - COSTA, G. S. Extração de espécies metálicas de resíduo sólido proveniente da pirólise de borra de petróleo.Universidade Federal do Paraná, Curitiba, 2010.

4 - CASTRO, R. A. Otimização do Sistema de Landfarming da RPBC - Refinaria Presidente Bernardes. Salvador, 2005.

5 - CARVALHO, D. D. Resíduos Sólidos. Universidade Federal do Rio de Janeiro, RJ.

6 - PASSOS, A. L. Tratamento avançado de resíduos sólidos perigosos: a experiência da cetrel. Salvador.

7 - CONEGLIAN, C. M. R. et al. Avaliação da biodegradação no solo de resíduos gerados em refinaria de petróleo. Rio de Janeiro, 2005.

8 - HENCKLEIN, F. A. et al. Avaliação da biodegradação de resíduos de hidrocarbonetos no solo de "landfarming" utilizando espectroscopia de absorção ótica no infrevermelho. São Paulo, 2007.

9 - CASTRO, R. A. Tratamento de Lodo de esgoto urbano no solo com microrganismos de "landfarming" e substrato oleoso. Universidade Federal do Paraná, 2000.

10 - VIANA, F. V. Acumulação de HPAs pela spartina alterniflora cultivada em solo de landfarming de indústria petroquímica. Universidade federal do Rio Grande, Rio Grande, 2008.

11 - ABNT - Associação Brasileira de Normas Técnicas: NBR 13894. Rio de Janeiro, 1997.

12 - SILVA, E. L.; MENEZES, E. M. Metodologia da Pesquisa e Elaboração de Dissertação. Florianópolis, 2005. 
13 - LEVENSPIEL, O. Engenharia das Reações Químicas. 2ed. Ed. Edgard Blucher. 2008.

14 - SILVA, L. J. Processo de Landfarming par tratamento de resíduos oleosos. UFRJ, Rio de Janeiro, Junho de 2009.

15 - UMITCH. Disponível em: http://www.umich.edu/ elements/course/lectures/two/index.htm

16 - TOCHETTO, M. R. L. Gerenciamento de resíduos sólidos industriais. Universidade Federal de Santa Maria, 2005.

17 - GUIMARAES, A. K. V. Extração do óleo e caracterização dos resíduos da borra de petróleo para fins de reuso. Universidade Federal do Rio Grande do Norte, Rio de Janeiro, Junho de 2007.

18 - OLIVEIRA, D. M. Aplicação da técnica de solidificação/estabilização para resíduos oleosos da indústria petrolífera, utilizando solo argiloso e bentonita. Universidade Federal de Santa Catarina, Florianopolis, Abril de 2003.

19 - NAPOLEÃO, D. C. et al., Estudo do processo Foto-Fenton para tratamento de fármacos: otimização e modelagem cinética. Scientia Plena 9, 094201 (2013).

20 - NUNES, C. A. Sistema de tratamento e destinação final de resíduos sólidos industriais gerados na indústria de produção de insulina. $20^{\circ}$ Congresso Brasileiro de Engenharia Sanitária e Ambiental, Belo Horizonte.

21 - SILVA, A. B. Comportamento dos setores químicos e petroquímico com relação aos passivos ambientais e os problemas causados em torno da Baía de Guanabara Escola nacional de saúde publica da fundação Oswaldo. Rio de Janeiro, 2001. 